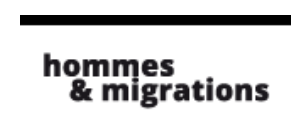

Hommes \& migrations

Revue française de référence sur les dynamiques

migratoires

$1284 \mid 2010$

Migrations et environnement

\title{
Île d'Hispaniola. Des voisins inégaux confrontés à des défis semblables : dégradation environnementale et migration
}

\section{Stefan Alscher}

Traducteur : Françoise Bouillot

\section{(2) OpenEdition}

\section{Journals}

Édition électronique

URL : http://journals.openedition.org/hommesmigrations/1248

DOI : 10.4000/hommesmigrations. 1248

ISSN : 2262-3353

\section{Éditeur}

Musée national de l'histoire de l'immigration

Édition imprimée

Date de publication : 1 mars 2010

Pagination : 84-96

ISSN : 1142-852X

Référence électronique

Stefan Alscher, « Île d'Hispaniola. Des voisins inégaux confrontés à des défis semblables : dégradation environnementale et migration », Hommes \& migrations [En ligne], 1284 | 2010, mis en ligne le 29 mai 2013, consulté le 01 mai 2019. URL : http://journals.openedition.org/hommesmigrations/1248 ; DOI $10.4000 /$ hommesmigrations. 1248 


\section{Île d'Hispaniola Des voisins inégaux confrontés à des défis semblables : dégradation environnementale et migration}

Par Stefan Alscher, chercheur au Centre pour la migration, la citoyenneté et le développement (Comcad), Université de Bielefeld, Allemagne

La République dominicaine et Haïti, qui partagent la même île et donc le même écosystème, sont confrontés aux mêmes défis environnementaux. L'île d'Hispaniola étant située sur le passage de tempêtes tropicales, les deux pays sont exposés aux catastrophes naturelles, aggravées par l'action des hommes. En Haïti, la quasi-totalité des forêts a disparu. Du côté dominicain,

le problème de la déforestation et de l'érosion des sols se pose également, mais dans une moindre mesure. Cette recherche, antérieure au séisme qui a durement frappé Haïti début 2010, contribue à déterminer le poids de la dégradation environnementale dans les flux migratoires sur l'île d'Hispaniola. 
Lîle d'Hispaniola, la seconde par la taille et la plus peuplée de la mer des Caraïbes, est partagée entre deux États indépendants : à l'ouest, Haïti, où l'on parle français et créole, à l'est, la République dominicaine, où l'on parle espagnol. D'un côté de l'île se trouve le pays le plus pauvre de l'hémisphère Nord (Indice de développement humain [IDH] de $0,475,154^{\mathrm{e}}$ rang), et de l'autre, un pays au revenu moyen-faible (IDH de 0,749), nouveau paradis touristique aux stations bien équipées et aux plages de sable blanc. Il n'est donc pas surprenant que le secteur des services, essentiellement représenté par le tourisme, soit la principale source de revenus de la République dominicaine, générant près de 60 \% de son PIB. En Haïti, le secteur agricole composé en majorité de petites exploitations de subsistance - ne représente que $30 \%$ du PIB, mais reste la principale source de revenus pour $70 \%$ de la population. Selon l'ONG Social Watch, 78 \% de la population haïtienne vit avec moins de 2 dollars par jour (16\% en République dominicaine) et près de la moitié (47\%) souffre de malnutrition ( 27 \% en République dominicaine $)^{(1)}$. Les deux pays partagent le même écosystème et affrontent donc des défis environnementaux comparables, voire semblables. L'île étant située sur le trajet des tempêtes tropicales, elle est exposée à de fortes précipitations et inondations pendant la saison des ouragans.

Les ressources forestières ont souffert, de part et d'autre, d'une déforestation et d'une érosion des sols à grande échelle, mais à des degrés différents. L'hypothèse centrale de cette contribution est que la dégradation environnementale est un facteur intensifiant de la migration interne et internationale. Une hypothèse secondaire est que les politiques adoptées jouent un rôle crucial dans la maîtrise, le ralentissement ou même l'inversion des processus de dégradation, réduisant ainsi les leviers de la migration comme stratégie d'adaptation.

\section{Une migration sous pression}

Haïti est un cas très souvent cité dans les études sur la dégradation environnementale. Presque toutes ses forêts ont disparu et une partie importante de son territoire souffre d'une grave érosion des sols qui menace les moyens de subsistance de la population. Selon Jacobson, les premières références aux boat people haïtiens en tant que "réfugiés environnementaux" remontent à $1984^{(2)}$. Pour Jacobson, leur migration est pour l'essentiel induite par l'environnement, mais Barker critique l'usage du terme de "réfugiés environnementaux" comme imprécis et introduit une série d'autres facteurs qui poussent les Haïtiens à quitter leur pays. Les deux auteurs s'accordent à dire que les catastrophes naturelles en Haïti sont aggravées par les activités humaines, qui réduisent encore "la capacité des écosystèmes à se régénérer" 
"catastrophes non naturelles (4)". Myers souligne sans mâcher ses mots que les Haïtiens "abandonnent leur terre natale notamment parce que leur pays est devenu une poubelle environnementale ${ }^{(5)}$ ". Pour lui, les Haïtiens n'ont pas seulement fui l'oppression politique, mais ont aussi été "poussés à partir par l'épuisement à grande échelle des ressources environnementales - sol, eau et arbres - qui forment la base de leur économie agricole $^{(6)}$ ". Il en conclut que la pression environnementale est le facteur prédominant de la migration des Haïtiens. Dans une étude pour le Natural Heritage Institute, Leighton-Schwartz et Notini se montrent plus sceptiques, soutenant que les facteurs environnementaux ne jouent qu'un rôle mineur dans une migration essentiellement politique et économique ${ }^{(7)}$. Catanese souligne que la migration des Haïtiens relève des deux phénomènes : de facteurs environnementaux et politiques qui "se sont renforcés l'un l'autre sur de longues périodes de l'histoire haïtienne ${ }^{(8) " . ~ I l ~}$ rappelle également que la faiblesse de l'économie haïtienne est aggravée par la déforestation et l'érosion des sols.

L'île de Saint-Domingue (Hispaniola) a été la première implantation espagnole permanente dans le Nouveau Monde. Une économie de plantations fut instaurée et les esclaves africains remplacèrent la population autochtone, affaiblie par les maladies et les massacres. En 1697, la partie occidentale de l'île fut cédée à la France et, un siècle plus tard, l'île entière devint territoire français par le Traité de Bâle. Les décennies suivantes furent caractérisées par des luttes pour l'indépendance, que la partie occidentale de l'île (Haïti) obtint en 1804, déclarant l'abolition de l'esclavage

\section{Les problèmes majeurs de l'île sont la déforestation, l'érosion des sols et des ressources insuffisantes en eau potable.} pour la première fois dans le monde. Les deux tiers orientaux de l'île finirent par gagner leur indépendance en 1863, après avoir subi la domination d'Haïti, connu maints troubles politiques, et accepté un statut colonial à court terme. Au début du $\mathrm{XX}^{\mathrm{e}}$ siècle, les deux parties de l'île subirent l'occupation des troupes américaines, suivie de deux régimes dictatoriaux : le général Trujillo dirigea la République dominicaine de 1930 à 1961, tandis que les Duvalier ("Papa Doc" et "Baby Doc") instauraient un régime de terreur de 1957 à 1986, appuyés par les Tontons macoutes de sinistre mémoire et leurs milices paramilitaires. Haïti ne connaît toujours pas la paix, ces vingt dernières années ayant également été marquées par de violents conflits, comme le coup d'État contre Aristide au début des années quatre-vingt-dix et les émeutes de la faim en 2008, qui ont conduit à la démission du Premier ministre, Jacques Édouard Alexis. Après le second mandat de Balaguer (de 1986 à 1996), qui s'était maintenu au 
pouvoir grâce à la fraude électorale, le système politique de la République dominicaine semble aujourd'hui plus stable, avec son alternance entre deux partis dominants (le PLD et le PRD).

\section{Les problèmes environnementaux d'Hispaniola}

L'histoire de la colonisation, des régimes répressifs successifs et des troubles politiques permanents dans les deux pays n'est pas étrangère aux phénomènes actuels de dégradation environnementale. Les problèmes majeurs de l'île sont la déforestation, l'érosion des sols et des ressources insuffisantes en eau potable. Sur l'Environmental Sustainability Index 2005 (devenu Indice de durabilité environnementale en 2006), Haïti est au $141^{\circ}$ rang sur 146 pays, alors que la République dominicaine est au $119^{\circ}$ rang, juste devant Haïti et Trinidad et Tobago pour les Amériques. C'est un net indicateur de l'impact des problèmes environnementaux dans les deux parties de l'île. La déforestation en Haïti a commencé dès les XVII et $\mathrm{XVIII}^{\mathrm{e}}$ siècles, quand les colons espagnols, puis français, ont abattu les forêts pour l'économie de plantation et la production de bois. Avec l'indépendance du pays, la situation empira encore : en 1825, l'élite politique d'Haïti convint avec l'ancienne puissance coloniale d'une dette à payer pour cette indépendance, avec la promesse d'être reconnue comme membre de la communauté internationale. Les paiements de réparation ont été estimés à 21 milliards de dollars actuels et ont été versés jusqu'en $1947^{(9)}$. Pour payer leur dette, les Haïtiens coupèrent leurs forêts et vendirent les bois tropicaux pour la production de meubles. Mais la déforestation continua de se poursuivre, même une fois la dette remboursée, l'abattage des arbres servant cette fois à la production de charbon de bois et au défrichement. En 1950, un quart d'Haïti était encore couvert de forêts. À la fin des années quatre-vingt, elles ne couvraient plus que $10 \%$ du territoire, pour atteindre $4 \%$ seulement en 1994. Aujourd'hui, la forêt ne compte plus que pour $1,4 \%$ du territoire d'Haïti ${ }^{(10)}$.

Dans le cas de la République dominicaine, l'expansion des plantations agricoles ainsi que l'usage de charbon de bois comme source d'énergie a réduit la couverture boisée d'environ $75 \%$ en $1922^{(11)}$ à $12 \%$ au début des années quatre-vingt, pour remonter à 28 \% de nos jours. L'accroissement de la couverture boisée en République dominicaine depuis les années quatre-vingt est dû aux programmes de reforestation à grande échelle (Plan Quisqueya Verde) et à la promotion d'autres sources d'énergie que le charbon de bois ${ }^{(12)}$. Dans son livre sur l'effondrement des civilisations, Jared Diamond oppose les problèmes environnementaux d'Haïti à ceux de son voisin. 
Pour lui, les limites environnementales expliquent en partie la pauvreté d'Haïti : "Un pourcentage plus élevé de la surface d'Haïti est montagneux, la zone de plaine propre à l'agriculture intensive est bien plus restreinte, il y a davantage de terrain calcaire, les sols sont plus fins, moins fertiles, et dotés d'une moindre capacité à récupérer ${ }^{(13)}$."

Conséquence de la déforestation à grande échelle, l'érosion des sols est devenue un problème majeur en Haïti. Selon des estimations des Nations unies, environ 50 \% de l'humus a été emporté par l'océan, et les terres affectées sont devenues impropres à l'agriculture ${ }^{(14)}$. L'érosion des sols suscite des problèmes environnementaux en chaîne tels que la réduction des terres agricoles, la réduction du taux d'infiltration des eaux dans les couches souterraines, la sédimentation des plaines, l'obstruction des systèmes de drainage et l'accumulation de sédiments dans les zones côtières ${ }^{(15)}$.

\section{Déforestation, érosion, inondations : l'équation du désastre}

La disparition des forêts d'Haïti a entraîné une modification du régime des précipitations, avec une probabilité accrue de sécheresse. Lors de pluies diluviennes, surtout au cours de la saison des ouragans, l'absence de barrières naturelles favorise les coulées de boue, qui ont enfoui à plusieurs reprises des villages entiers. La plus grande vulnérabilité d'Haïti devient évidente quand on compare l'impact des tempêtes tropicales et d'autres événements climatiques extrêmes en Haïti et en République dominicaine. Quand l'ouragan Jeanne est passé sur l'île d'Hispaniola en septembre 2004, plus de 3000 personnes ont trouvé la mort en Haïti, alors que la République dominicaine n'avait à déplorer la perte que de 19 citoyens, la plupart emportés par les rivières en crue.

Tableau 1. Conséquences des cinq catastrophes naturelles majeures sur l'ile d'Hispaniola (1900-2008)

\begin{tabular}{|c|c|c|c|c|c|c|c|c|}
\hline \multirow[b]{2}{*}{$\begin{array}{l}\text { Type } \\
\text { d'événement }\end{array}$} & \multicolumn{2}{|c|}{$\begin{array}{l}\text { Nombre } \\
\text { d'événements }\end{array}$} & \multicolumn{2}{|c|}{$\begin{array}{l}\text { Nombre } \\
\text { de morts }\end{array}$} & \multicolumn{2}{|c|}{$\begin{array}{c}\text { Total des gens } \\
\text { affectés }\end{array}$} & \multicolumn{2}{|c|}{$\begin{array}{c}\text { Dommages } \\
\text { matériels } \\
\text { en \$ US }\end{array}$} \\
\hline & $\begin{array}{l}\text { Rép. } \\
\text { Dom. }\end{array}$ & Haîti & $\begin{array}{l}\text { Rép. } \\
\text { Dom. }\end{array}$ & Haïti & $\begin{array}{l}\text { Rép. } \\
\text { Dom. }\end{array}$ & Haiiti & $\begin{array}{l}\text { Rép. } \\
\text { Dom. }\end{array}$ & Haîti \\
\hline Tempête & 20 & 26 & 4.462 & 13.327 & 2.748 .059 & 2.926 .423 & 2.767 .910 & 982.906 \\
\hline Inondation & 17 & 41 & 844 & 4.995 & 1.467 .040 & 2.195.998 & 45.220 & 51.959 \\
\hline sécheresse & 1 & 7 & - & - & 240.000 & 2.305 .217 & 5.000 & 1.000 \\
\hline $\begin{array}{l}\text { Tremblement } \\
\text { de terre }\end{array}$ & 2 & 1 & 76 & 6 & 2.015 & - & - & 20.000 \\
\hline $\begin{array}{l}\text { Glissement } \\
\text { de terrain }\end{array}$ & - & 2 & & 262 & - & 1.060 & - & - \\
\hline
\end{tabular}


Le tableau 1 indique le nombre de morts et de gens affectés par les catastrophes naturelles sur l'île d'Hispaniola, ainsi que le montant des dommages matériels. Devant ces chiffres, il devient évident que la partie haïtienne de l'île tend à avoir un plus grand nombre de morts ou de gens affectés que la République dominicaine. Du fait de la déforestation à grande échelle et de l'érosion des sols, les inondations, comme les sécheresses, sont plus fréquentes en Haïti et ont un plus grand impact que chez son voisin.

Autre exemple transfrontalier des impacts des événements climatiques extrêmes, en mai 2004, après de violentes précipitations (jusqu'à 500 mm), des crues subites et des glissements de terrain ont affecté certains quartiers des villes de Jimaní (République dominicaine) de Mapou et de Fonds-Verrettes (Haïti). Environ 700 personnes furent tuées en République dominicaine, alors qu'Haïti déplorait la mort de plus de 2600 habitants. Un total de 41300 personnes furent affectées par les inondations ${ }^{(16)}$. Le principal facteur de cette intense destruction était la déforestation à grande échelle dans les bassins de drainage et la présence d'installations humaines dans les plaines fluviales et autres zones basses du flanc sud du Massif de la Salle.

Les impacts des inondations sont aggravés également par l'extraction d'agrégats sur

Le développement immobilier sauvage, surtout dans les zones urbaines et, pour la République dominicaine, dans les zones touristiques, a conduit à de sévères problèmes d'approvisionnement en eau, aggravés encore au cours de la saison sèche. les rives des fleuves. Ces agrégats - en général des pierres et du calcaire - sont surtout utilisés comme matériaux de construction. Selon José Contreras, de l'Instituto Tecnológico de Saint-Domingue (INTEC), le nombre de rivières naturelles en République dominicaine a décru de $70 \%$, du fait de l'extraction d'agrégats. Les anciens cours d'eau sont devenus de petits ruisseaux ou ont totalement disparu. En cas de fortes précipitations, les masses d'eau tendent à se répandre à l'horizontale, les anciens lits des rivières ayant disparu ${ }^{(17)}$.

Même si cela peut sembler paradoxal à première vue, les pénuries d'eau sont un autre problème environnemental affectant les deux côtés de l'île. Le développement immobilier sauvage, surtout dans les zones urbaines et, pour la République dominicaine, dans les zones touristiques, a conduit à de sévères problèmes d'approvisionnement en eau, aggravés encore au cours de la saison sèche. Dans certaines régions de la République dominicaine, des usines de dessalement d'eau de mer ont été installées pour éviter l'intrusion de l'eau de mer dans la nappe phréatique naturelle. La contamination industrielle est un autre facteur responsable de la mauvaise qualité de l'eau. La République dominicaine a connu une croissance industrielle 
incontrôlée au cours des quarante dernières années, en l'absence de tout programme de production propre ou de contrôle de la contamination.

Enfin, la côte est menacée d'une part, par l'érosion des plages, et d'autre part, par le glissement du sol érodé dans la mer, qui endommage les récifs de corail. Ce sont là de graves défis pour l'avenir, si l'on tient compte de l'importance du tourisme, l'une des sources de revenus les plus importantes de l'économie dominicaine.

\section{Revue d'ensemble des processus de migration}

Depuis le début du XX $\mathrm{XX}^{\mathrm{e}}$ siècle, la République dominicaine a employé de la maind'ceuvre haïtienne, surtout dans les champs de canne à sucre. Mais malgré une longue tradition de migration, les Haïtiens en République dominicaine ont été et sont encore confrontés aux préjugés, voire à la violence. Parmi ces épisodes, le massacre Perejil ${ }^{18)}$ en 1937, quand 17000 à 35000 Haïtiens furent massacrés à la frontière par des soldats et les policiers dominicains, pèse encore à ce jour sur les rapports entre les deux États. Les soldats agissaient sur ordre du dictateur Trujillo, qui agitait la menace d'une inva-

\section{Contrairement aux Cubains, les migrants haïtiens sont en général déportés immédiatement s'ils sont repérés par les services d'immigration des États-Unis, mais beaucoup tentent à nouveau leur chance, en transitant d'abord par d'autres îles comme les Bahamas.} sion pacifique par les immigrés haïtiens dans une tentative de regagner le contrôle de l'île tout entière.

La construction d'un scénario d'invasion où les immigrés haïtiens seraient une menace constante est un leitmotiv de la politique dominicaine, qui sert surtout à dissimuler les problèmes internes et à renforcer l'identité nationale. En même temps, un antihaïtianisme largement répandu, ancré dans l'occupation haitienne de la première moitié du XIX ${ }^{e}$ siècle, ainsi que des politiques de restriction de l'immigration et des campagnes de déportation permettent de maintenir de bas salaires pour la main-d'ceuvre haïtienne migrante. Le nombre total d'Haïtiens vivant de façon temporaire ou permanente en République dominicaine est en général exagéré pour des raisons idéologiques, avec des chiffres variant de 1 à 2 millions de migrants. Les spécialistes des migrations avancent toutefois une estimation qui ne dépasse pas 250000 à 500000 immigrés ${ }^{(19)}$. Les preuves présentées au Groupe de travail des Nations unies sur l'esclavage, et les rapports de diverses organisations des droits de l'homme révèlent que les salaires sont misérablement bas et que les conditions de travail et d'existence ne répondent pas aux critères posés par les deux ${ }^{200}$. 


\section{Hailti, pays d'émigration économique}

Dans l'histoire de la migration haïtienne, ce sont surtout les facteurs politiques et économiques - imbriqués dans les problèmes de dégradation environnementale qui ont été et sont encore les principaux leviers de la migration interne et internationale. La première migration de masse en Haïti (urbaine > rurale) est survenue peu après l'indépendance, dans le cadre de la réforme agraire postcoloniale et de la décentralisation des pouvoirs des centres urbains vers les zones rurales $^{(21)}$. De la fin du XIX ${ }^{\mathrm{e}}$ siècle aux années trente, le pays a connu une autre vague de migration, cette fois d'Haïtiens ruraux émigrant vers Cuba et vers la République dominicaine pour travailler dans les plantations de canne à sucre. Les flux de migration internationale vers les États-Unis ont commencé dans les années cinquante, impliquant cette fois des Haïtiens dotés d'un bon niveau d'éducation en quête d'un meilleur niveau de vie à l'étranger. Cette "fuite des cerveaux" n'a fait que s'accroître sous le régime dictatorial des Duvalier (1957 à 1986). À partir des années soixante et jusqu'aux années quatre-vingt, des Haïtiens semi qualifiés des classes urbaines moyennes et inférieures se sont joints à ce flux. Dans les années soixante, l'émigration a encore été aggravée par une crise économique et par les impacts de l'ouragan Flora, qui fit plus de 5000 morts en octobre $1963^{(22)}$.

À partir des années soixante-dix, ceux qui n'avaient pas les moyens d'acheter un visa ou un billet d'avion ont tenté d'atteindre les rivages du sud de la Floride par bateau. Ces boat people étaient plus souvent issus de villes et de villages que de Port-auPrince. La pauvreté et la répression croissante des Tontons macoutes ont accru cet exode par la mer. Ce flux cessa un court moment en 1990 avec l'élection d'Aristide à la présidence, qui souleva l'espoir d'un développement paisible. Mais sous la junte militaire de 1991 à 1994, des milliers d'Haïtiens quittèrent à nouveau leur pays, essentiellement par bateau. La surpopulation et le désespoir ont nourri l'exode hors d'Haïti. En 2003, les garde-côtes américains ont arrêté 2000 boat people haïtiens qui tentaient d'atteindre les rives américaines - le chiffre le plus élevé de toutes les Antilles. Contrairement aux Cubains, les migrants haïtiens sont en général déportés immédiatement s'ils sont repérés par les services d'immigration des États-Unis, mais beaucoup tentent à nouveau leur chance, en transitant d'abord par d'autres îles comme les Bahamas. De nos jours, des millions d'Haïtiens vivent à l'étranger et ont constitué d'importants réseaux entre les régions d'origine et de destination, principalement aux États-Unis, au Canada (surtout au Québec), en République dominicaine et en France, mais aussi aux Bahamas, à Cuba et dans les Petites Antilles. On trouve d'importantes communautés de Haïtiens à Miami ("Little Haïti”) et à New York (Brooklyn et Queens). 


\section{Les destinations des migrants dominicains}

En République dominicaine, la migration internationale est souvent liée à la migration interne. Le modèle de migration typique commence par une migration rurale > urbaine, surtout vers les deux centres urbains - Saint-Domingue et Santiago -, mais aussi vers les capitales provinciales et les régions de tourisme international, comme Puerto Plata ou La Romana. L'une des causes principales de l'exode rural est la crise de l'agriculture, marquée par des prix en baisse constante, par l'abandon des zones rurales de la part du gouvernement et, dans certaines régions, par des problèmes de dégradation environnementale. Les centres urbains servent de tremplins pour la migration internationale. En ville, les migrants sont en mesure de prendre des contacts et de gagner la somme nécessaire pour payer leur passage (et parfois leur visa).

La migration internationale des Dominicains vers les États-Unis (mais aussi vers d'autres destinations, comme l'Espagne) a commencé au début des années soixante après l'assassinat du dictateur Trujillo. Un fort chômage et une forte répression politique ont conduit à une nouvelle émigration entre 1966 et 1978. Ce groupe d'émigrants a contribué à établir un réseau pour les nouveaux arrivants. Une troisième vague d'émigration, due à la récession économique, a commencé au début des années quatre-vingt. Outre les États-Unis et le Canada, l'Espagne et l'Italie (et dans une moindre mesure d'autres pays européens) sont devenus d'importantes destinations pour les migrants dominicains. La migration dominicaine vers l'Espagne présente des traits particuliers, comme la prédominance des femmes et le caractère domestique de la main-d'ceuvre ${ }^{(23)}$.

Selon les données de l'état civil espagnol local (padrón municipal), environ 65000 citoyens dominicains vivaient en Espagne en 2007, chiffre qu'il faut rapporter aux 25000 recensés en 2000, installés pour la plupart dans les zones urbaines de Madrid (40\%) et de Barcelone $(22 \%)^{24)}$. Des données de l'ambassade espagnole à Saint-Domingue montrent aussi un net accroissement de la migration vers l'Espagne: si le consulat délivrait environ 16500 visas pour les citoyens dominicains en 2004, ce chiffre est monté à 20000 en 2005 et 2006, pour atteindre un nouveau pic de 24356 visas délivrés en 2007 $7^{(25)}$. L'émigration dominicaine vers l'Espagne provient surtout du sud-ouest de la République dominicaine (municipalités de Vicente Noble, Tamayo, Neyba, Postrer Río, La Descubierta, Jimaní, etc.). Aux États-Unis, la communauté migrante dominicaine se concentre surtout à New York et dans le New Jersey, mais aussi en Floride (Miami/Broward County). 


\section{Travail de terrain, résultats et analyses}

Le travail de terrain sur l'île d'Hispaniola (mené entre février et mars $2008^{(26)}$ ) s'est essentiellement fondé sur des entretiens avec des experts d'une part, et des entretiens avec des habitants des zones affectées d'autre part.

En Haïti, l'étendue de la catastrophe environnementale devient manifeste dans les entretiens qualitatifs avec les gens vivant dans les zones affectées (un total de dix). Parmi les affirmations typiques: "Nous ne pouvons pas cultiver ici parce que le sol n'est que du sable", "L'eau pénètre dans la maison quand il pleut", ou même "Quand le cyclone est passé, plus de 24 maisons de notre quartier ont disparu." Ces trois phrases reflètent les principaux problèmes environnementaux d'Haït : des terres rendues infertiles par la déforestation et l'érosion des sols, des inondations au cours de la saison des pluies, et une forte vulnérabilité au passage des ouragans et autres tempêtes tropicales. Néanmoins, seuls quatre interviewés sur dix envisagent actuellement de migrer ou perçoivent la migration comme une option pour améliorer leurs moyens d'existence. Les six autres n'envisagent pas la migration comme une option viable. Les liens familiaux et la "foi en Dieu" sont parmi les raisons mentionnées pour rester sur le lieu de résidence, malgré les problèmes rencontrés par les interviewés.

En République dominicaine - ou pour être plus précis, dans les communautés frontalières analysées près de La Descubierta et Neyba - la déforestation, l'érosion des sols qui en résulte, ainsi que les inondations et les glissements de terrain au cours des fortes intempéries sont mentionnés comme des facteurs contribuant à la décision de migrer. Mais dans la plupart des cas, ils sont perçus comme des facteurs de pression supplémentaires plutôt que comme le facteur premier. Les problèmes les plus graves demeurent l'absence de services sociaux (écoles, services de santé, etc.) et l'abandon du secteur agricole de la part du gouvernement (suppression de subventions, etc.). Sept interviewés sur dix voyaient l'émigration comme une option viable, en soulignant que jusqu'à présent, l'opportunité de partir ne s'était pas présentée.

\section{Migration environnementale : le choc des cultures}

Le "point de rupture" serait pour la plupart la survenue d'une catastrophe naturelle comme une longue sécheresse ou un ouragan. Les experts dominicains en études environnementales et en recherche sur les migrations s'accordent à dire que les facteurs environnementaux jouent bien un rôle dans la migration, surtout du fait de 
l'impact de la dégradation environnementale sur l'agriculture des petits exploitants, base vivrière d'une grande part de la population dans les régions rurales. Le SudOuest dominicain est l'une des zones les plus affectées. Lorsqu'on la compare à des régions dotées de terres plus fertiles, l'impact sur la migration devient évident ${ }^{(27)}$. La migration interne et internationale étant essentiellement féminine dans le cas de la République dominicaine, certains chercheurs observent "une expulsion des femmes des zones rurales", celles-ci allant chercher une alternative dans les services domestiques et étant donc contraintes de migrer vers les centres urbains, voire à l'étranger ${ }^{(28)}$. La monoculture agro-industrielle exerce une pression sur les systèmes traditionnels (et plus durables) de production agricole ${ }^{(29)}$. Elle emploie une maind'œuvre plutôt masculine que féminine, contraignant les femmes à quitter leur région d'origine.

Une particularité inquiétante de la zone frontalière dominicaine-haïtienne est l'existence de forts préjugés contre les migrants haïtiens. De nombreux villageois dans la région étudiée accusaient les migrants haïtiens d'être responsables de la déforestation et de l'érosion des sols par l'application de leur modèle traditionnel d'"essartage" avant la culture sur brûlis, qui consiste à abattre les arbres pour dégager de la terre cultivable. Certains villageois ont même mentionné la probabilité de violents conflits à l'avenir. Ils ne mentionnaient pas toutefois que les émigrants dominicains issus de ces mêmes villages pratiquent une forme de métayage, en prêtant leurs terres aux migrants haïtiens et en tirant profit de cet usage de la terre par le partage du revenu des récoltes. En outre, des experts des rapports dominicainshaïtiens soulignent que les manifestations d'anti-haïtianisme envers les "étrangers" sont une caractéristique de la zone frontalière, alors que dans la vie quotidienne, les Dominicains et les Haïtiens vivent en paix, ce qui est notamment démontré par l'existence de mariages mixtes dans cette région ${ }^{(30)}$.

\section{Conclusion}

Si le lien entre dégradation environnementale et processus de migration est très évident dans le cas haïtien, il est plus complexe dans le cas dominicain. Le fort degré de déforestation, d'érosion des sols et de dégradation des terres en Haïti influence fortement les décisions de migration, la subsistance d'une grande part de la population se trouvant menacée, voire complètement détruite. En République dominicaine, on observe les mêmes problèmes de dégradation environnementale, bien qu'à un degré moindre. Des facteurs autres qu'environnementaux jouent aussi un rôle important dans la décision de migrer. Le cas dominicain montre en outre que l'impact des 
décisions politiques est non négligeable, les programmes de reforestation ayant réussi à récupérer de grandes étendues de forêts autrefois perdues. Néanmoins, cette étude de cas n'est qu'un instantané des problèmes existants. De nouvelles recherches sont nécessaires pour une analyse plus approfondie du lien complexe entre problèmes environnementaux, conflits et migration sur l'île d'Hispaniola.

\section{Article traduit par Françoise Bouillot}

\section{Notes}

1. Social Watch, Country by Country [Domincan Republic; Haïti], 2008.

<http://www.socialwatch.org/en/fichasPais/fichaPaisPortada.htm>, 22 juillet 2008.

2. J. Jacobson, "Environmental Refugees: A Yardstick of Habitability", Worldwatch Paper n86, Worldwatch Institute, Washington D.C, 1988.

3. D. Barker, "Environmental Migrants", Draft Paper for World Resources 1990-1991, 31 août 1989.

4. J. Jacobson, "Environmental Refugees:aa Yardstick of Habitability", op. cit.

5. N. Myers, Ultimate Security: The Environmental Basis of Political Stability, New York \& Londres, W.V. Norton, 1993. Nasa, Haitian Deforestation, Nasa Scientific Visualization Studio, 23 juillet 2008. $<$ http://svs.gsfc.nasa.gov/goto?2640>

6. N. Myers, "Environmental Refugees: A Growing Phenomenon of the 21st Century", in Proceedings of the Royal Society of London, n³57, 2001, p. 610.

<http://journals.royalsociety.org/content/rbaxblphf3rxdpcf/fulltext.pdf>

7. M. Leighton-Schwartz, J. Notini, "Preliminary Report on Desertification and Migration: Case Studies and Evaluation", in M. Puigdefábrigas (dir), Desertification and Migrations, Logroño/Spain, Geoforma Ediciones, 1995, p. 88 .

8. A. Catanese, Haitians - Migration and Diaspora, Boulder, Westview Press, 1999.

9. S. Johnson, "Extreme Events and Haitian Environmental Refugees", in ICE Case Studies, n 209, 23 juillet 2008, p. 4. <http://www.american.edu/ted/ice/haiti-hurricane.htm>

10. Jamaican Observer, $23 / 09 / 2004$.

11. R. Yunén, "Medio Ambiente en Haiti y la República Dominicana", in R. Silié, O. Inoa, A. Arnold (dir), La República Dominicana y Haití frente al Futuro, Saint-Domingue, FLACSO, 1998, p. 157.

12. Entretien avec José Contreras, INTEC, Saint-Domingue, 17/03/2008.

13. Jared Diamond, Collapse: How Societies Choose to Fail or Succeed, Londres \& New York, Penguin, 2005, p. 339.

14. T. Homer-Dixon, "Environmental Scarcities and Violent Conflict: Evidence from Cases", in International Security, vol. 19, n 1, 24 juillet 2008, pp. 5-40, <http://www.library.utoronto.ca/pcs/evidence/evid3.htm> ; ISRIC, Global Assessment of Human-induced Soil Degradation (GLASOD), 25 juillet 2008, <http://www.isric.org/UK/About+ISRIC/Projects/Track+Record/GLASOD.htm>

15. R. Léger, "Los Desafíos Ecológicos en Haití hacia el Año 2000", in R. Silié, O. Inoa, A. Arnold (dir), La República Dominicana y Haití frente al Futuro, Saint-Domingue, FLACSO, 1998, p. 204. 
16. EM-DAT, The OFDA/CRED International Disaster Database, Bruxelles, Université catholique de Louvain, 22 juillet 2008. <http://www.emdat.be>

17. Entretien avec José Contreras, INTEC, Saint-Domingue, 17/03/2008.

18. Les gens à la peau sombre étaient contraints de prononcer le mot espagnol "perejil" (persil). Ceux qui n'arrivaient pas à prononcer le "r" espagnol étaient soupçonnés d'être des immigrés haïtiens et tués à coups de machettes.

19. R. Silié, "Aspectos Socio-Históricos sobre la Inmigración Haitiana a la República Dominicana", in R. Silié, O. Inoa, A. Arnold (dir), La República Dominicana y Haití frente al Futuro, Saint-Domingue, FLACSO, 1998, p. 12.

20. Anti-Slavery, Forced Labour on Sugar Cane Plantations in the Dominican Republic, Groupe de travail sur les formes contemporaines de l'esclavage, $23^{\mathrm{e}}$ session, Genève, 25 juillet 2008,

http://www.antislavery.org/archive/submission/submission1998-05Dominican.htm ; HRW, "Illegal people: Haitians and Dominico-Haitians in the Dominican Republic", in Human Rights Watch, vol. 14, no. 1(B), 25 juillet 2008, http://www.hrw.org/reports/2002/domrep/.

Wooding, B.; Moseley-Williams, R. Inmigrantes Haitianos y Dominicanos de Ascendencia Haitiana en la República Dominicana, Saint-Domingue, CID et SJR, 24 juillet 2008.

$<$ http://espacinsular.org/IMG/_Inmigrantes_haitianos.pdf>

21. A. Catanese, A. Haitians - Migration and Diaspora, op. cit., p. 19.

22. Gordon E. Dunn, "The hurricane season of 1963", in Monthly Weather Review, vol. 92, n³, 24 juillet 2008, p. 134, http://docs.lib.noaa.gov/rescue/mwr/092/mwr-092-03-0128.pdf.

23. J. Romero, "La migración dominicana hacia españa, factores, evolución y desarrollo", in Revue européenne des migrations internationales, vol. 19, $\mathrm{n}^{\circ} 1,2007,<$ http://remi.revues.org/document $387 . \mathrm{html}$.

24. INEbase, Padrón Municipal: Explotación Estadística y Nomenclátor, 26 juillet 2008, http://www.ine.es/inebmenu/indice.htm\#6.

25. Entretien avec Manuel Hernández Ruigómez, consul général d’Espagne en République dominicaine, Saint-Domingue, 6 mars 2008.

26. Cette recherche a été menée dans le cadre du Projet EACH FOR avec le soutien financier du sixième programmecadre de la Commission européenne.

27. Entretien avec José Contreras, INTEC, Saint-Domingue, 17 mars 2008.

28. Entretien avec Fatima Portorreal, INTEC, Saint-Domingue, 6 mars 2008.

29. F. Portorreal, "Estructura Sociodemográfica de los Sectores Rurales Dominicanos", in PNUD, Nueva Ruralidad y Desarrollo Humano. La Nueva Ruralidad en República Dominicana: ¿Sigue siendo la Parte Atrás de lo Urbano o es una Oportunidad para el Desarrollo Humano?, Saint-Domingue, PNUD, 24 juillet 2008, pp. 69-82, http://pnud.sc17.info/files/forodh/Foro3-1.pdf.

30. Entretien avec Ruben Silié, FLACSO, Saint-Domingue, 17 mars 2008. 